\title{
ON OPERATOR BOHR TYPE INEQUALITIES
}

\section{LIMIN ZOU AND CHUANJIANG HE}

Abstract. The purpose of this paper is to discuss inequalities related to operator versions of the classical Bohr inequality. We obtain refinements of some inequalities due to Cheung and Pečarić [J. Math. Anal. Appl. 323 (2006) 403-412] and Zhang [J. Math. Anal. Appl. 333 (2007) 1264-1271]. Moreover, we present two inequalities for multiple operators, which are similar to ones proposed by Chansangiam et al. [J. Math. Anal. Appl. 356 (2009) 525-536].

Mathematics subject classification (2010): 47A63.

Keywords and phrases: Bohr inequality, operator inequalities, operator absolute value.

\section{REFERENCES}

[1] S. Abramovich, J. Barić, J. PeČArić, A new proof of an inequality of Bohr for Hilbert space operators, Linear Algebra Appl., Vol. 430, 4 (2009), p. 1432-1435.

[2] H. Bohr, Zur Theorie der Fastperiodischen Funktionen I, Acta Math., Vol. 45, 1 (1924), p. 29-127.

[3] P. Chansangiam, P. Hemchote, P. Pantaragrhong, Generalizations of Bohr inequality for Hilbert space operators, J. Math. Anal. Appl., Vol. 356, 2 (2009), p. 525-536.

[4] W. Cheung, J. PEČARIĆ, Bohr's inequalities for Hilbert space operators, J. Math. Anal. Appl., Vol. 323, 1 (2006), p. 403-412.

[5] M. FujiI, M. S. Moslehian, J. Mićić, Bohr's inequality revisited, Nonlinear analysis, Springer Optim. Appl. 68, 279-290, Springer, New York, (2012).

[6] M. FusII, H. Zuo, Matrix order in Bohr inequality for operators, Banach J. Math. Anal., Vol. 4, 1 (2010), p. 21-27.

[7] O. Hirzallah, Non-commutative operator Bohr inequality, J. Math. Anal. Appl., Vol. 282, 2 (2003), p. 578-582.

[8] J. S. Matharu, M. S. Moslehian, J. S. Aujla, Eigenvalue extensions of Bohr's inequality, Linear Algebra Appl., Vol. 435, 2 (2011), p. 270-276.

[9] M. S. Moslehian, R. RaJić, Generalizations of Bohr's inequality in Hilbert $C^{*}$-modules, Linear Multilinear Algebra., Vol. 58, 3 (2010), p. 323-331.

[10] F. Zhang, On the Bohr inequality of operators, J. Math. Anal. Appl., Vol. 333, 2 (2007), p. 1264 1271.

[11] L. Zou, C. HE, S. QAIS AR, Inequalities for absolute value operators, Linear Algebra Appl., Vol. 438, 1 (2013), p. 436-442. 